\title{
Utilidad de los diferentes métodos no invasivos de predicción de fibrosis hepática en pacientes del País Vasco con hemocromatosis fenotípica
}

\author{
A. Castiella, E. Zapata, P. Otazua, J. Fernández, J. M. Alustiza, M. Ugarte, L. Legasa, A. Galardi, \\ A. Ugalde, I. Barredo y J. A. Arriola \\ Servicios de Gastroenterología, Radiología y Anatomía Patológica. Hospitales de Mendaro, Mondragón y Donostia, \\ Osatek Donostia. Gipuzkoa
}

\section{RESUMEN}

Objetivo: determinar si el producto de la edad por la concentración de hierro hepático (índice de fibrosis) y los valores de plaquetas, ferritina y transaminasas están relacionados con el riesgo de padecer fibrosis avanzada $(F \geq 3)$ en hemocromatosis.

Métodos: estudio retrospectivo de 32 pacientes con hemocromatosis hereditaria con expresión fenotípica. Todos los pacientes fueron biopsiados obteniéndose la concentración de hierro hepático.

Resultados: en 7 pacientes se realizó RM $(1,5 \mathrm{~T})$ con obtención de concentración de hierro hepático (protocolo de Alustiza). Biopsia hepática: en 23 pacientes fibrosis 0-2; en 9 fibrosis 3-4. El índice de fibrosis mostró una especificidad del 68\%, sensibilidad del $85,7 \%$, VPP del 42,8\% y VPN del 94,4\% para fibrosis avanzada. La cifra de plaquetas $(<200.000)$ reveló un VPN 94,4\%, ferritina (> 1.000) VPN 75\% y el índice de fibrosis por RMN (punto corte 480.000) VPN 80\%. La combinación de los mismos, el índice de fibrosis (por biopsia o por RM) con las transaminasas y las plaquetas con las transaminasas, reveló un VPN del 100\%.

Conclusiones: el índice de fibrosis $(>480.000)$ y las plaquetas $(<200.000)$ tienen la mayor sensibilidad para predecir fibrosis de alto grado. Un resultado negativo en ambos permite descartar fibrosis significativa en el $94 \%$ de los casos. La RM permite una buena predicción de fibrosis.

Palabras clave: Hemocromatosis. Sobrecarga férrica. Resonancia magnética. Metabolismo férrico. Fibrosis hepática.

\begin{abstract}
Objective: to determine whether the product of multiplying age by liver iron concentration (LIC) (fibrosis index; cut-off, 480,000 ), platelets, transaminases, and ferritin values are related to the risk of high grade fibrosis.

Methods: a retrospective study of 32 patients with hereditary hemochromatosis $(\mathrm{HH})$ with phenotypic expression. All patients had a liver biopsy with LIC.

Results: in 7 patients a magnetic resonance imaging (MRI) scan $(1.5 \mathrm{~T})$ was obtained with LIC following Alustiza's protocol. Liver biopsy: fibrosis grade (F) $0-2$ in 23 patients; F 3-4 in 9. Fibrosis index (FI) showed a specificity of $68 \%$, sensitivity of $85.7 \%$, positive predictive value (PPV) of $42.8 \%$, and negative predictive value (NPV) of $94.4 \%$ for high-grade fibrosis. Platelet count $(<200,000)$ revealed a NPV of $94.7 \%$ for F3-4. Aspartate transaminase (AST) levels above the upper limit of normal showed a NPV of $94.4 \%$; ferritin levels (> 1.000) a NPV of $75 \%$, and MRI-derived LIC $x$ age $(>480,000)$ a NPV of $80 \%$. The combination of FI (either by biopsy or MRI) with transaminases, and of platelets with transaminases revealed a NPV of $100 \%$.

Conclusions: FI > 480,000 and platelets < 200,000 have the highest sensitivity for high-degree fibrosis prediction. A negative result allows to discard significant fibrosis in $94 \%$ of cases. MRI allows a good fibrosis prediction
\end{abstract}

Key words: Hemochromatosis. Iron overload. Magnetic resonance imaging. Iron metabolism. Liver fibrosis.

Castiella A, Zapata E, Otazua P, Fernández J, Alustiza JM, Ugarte M, Legasa L, Galardi A, Ugalde A, Barredo I, Arriola JA. Utilidad de los diferentes métodos no invasivos de predicción de fibrosis hepática en pacientes del País Vasco con hemocromatosis fenotípica. Rev Esp Enferm Dig 2008; 100: 611-614

\section{INTRODUCCIÓN}

La hemocromatosis hereditaria $(\mathrm{HH})$ es la enfermedad genética más frecuente en poblaciones de ascendencia

Recibido: 29-04-08

Aceptado: 08-07-08

Correspondencia: Agustín Castiella. Servicio de Gastroenterología. Hospital de Mendaro. Barrio Mendizábal, s/n. 20850 Mendaro.

e-mail: agustincastiella@yahoo.es europea $(1,2)$. En 1996 Feder y cols. (3) clonaron el gen HFE de pacientes con $\mathrm{HH}$, descubriendo dos mutaciones del gen que causa la enfermedad: C282Y y H63D. Esto ha sido de gran utilidad en el diagnóstico pero la prevalencia de estas mutaciones varía mucho según la localización geográfica, con un gradiente decreciente norte-sur (4). Estas mutaciones no están siempre asociadas con una expresión fenotípica de la enfermedad y, hoy en día, sigue siendo muy controvertido qué porcentaje de homocigotos desarrolla enfermedad clínica (5-7). 
La biopsia hepática, con estudio histológico y cuantificación de la concentración de hierro hepático $(\mathrm{CHH})$, ha sido el método estándar para el diagnóstico de la enfermedad, pero desde que disponemos de pruebas genéticas esto ha cambiado, utilizándose la biopsia habitualmente con fines pronósticos (8). Se recomienda realizar biopsia hepática en todos los pacientes salvo en aquellos que sean homocigotos $\mathrm{C} 282 \mathrm{Y}$ o heterocigotos $\mathrm{C} 282 \mathrm{Y} / \mathrm{H} 63 \mathrm{D}$, y que tengan unos niveles de ferritina sérica inferiores a $1.000 \mu \mathrm{g} / \mathrm{l}$, edad menor de 40 años y trasaminasas normales (5). Esto se basa en cuatro estudios clínicos (9-12) que han estudiado cuáles eran los factores predictivos no invasivos para fibrosis de alto grado o cirrosis en sus series clínicas. Más recientemente, Olynyk y cols. (13) han determinado que el tiempo de exposición al hierro por parte del hígado incrementa el riesgo de fibrosis significativa en HH. Esto se calculó mediante el producto de la $\mathrm{CHH}$ por la edad de los pacientes, con un punto de corte en 480.000 (si menor, fibrosis de bajo grado $-\mathrm{F} \leq 2-$ ).

El objetivo de nuestro estudio es determinar si estos predictores no traumáticos de fibrosis son útiles en nuestros pacientes.

\section{PACIENTES Y MÉTODOS}

\section{Pacientes}

Hemos revisado las historias clínicas de los pacientes con HH de dos hospitales (Mendaro y Mondragón, Gipuzkoa) que atienden a una población de 140.000 personas.

Se obtuvieron los siguientes parámetros clínico-analíticos: edad, sexo, ferritina pre-tratamiento, saturación de transferrina, hierro sérico, transaminasas, plaquetas, mutaciones HFE, coagulación, serología VHB y VHC, estadio de fibrosis en biopsia hepática (F0-4), RM con $\mathrm{CHH}$, índice de fibrosis (edad x $\mathrm{CHH}$ ).

Criterios de inclusion: hemocromatosis fenotípica, $\mathrm{CHH}>4.000$ o índice de hierro hepático $>1,9$; ausencia de sobrecarga secundaria de hierro hepático; disponibilidad de ferritina pre-tratamiento; ingesta etílica menor de $60 \mathrm{~g} / \mathrm{d}$ ía; ausencia de factores de riesgo o evidencia histológica de esteatohepatitis no alcohólica (IMC $<33$; triglicéridos $<500$, ausencia de diabetes mellitus tipo 2; exclusión de otras causas de enfermedad hepática $(10,12)$; biopsia hepática con tinción de hierro (tinción de Perls 3+ $\mathrm{o}>$ ) y gradiente periportal a pericentral (14).

\section{Métodos}

-Índice de hierro hepático: $\mathrm{CHH} / \mathrm{edad}$.

-Análisis mutación HFE (4): se realizó siguiendo método descrito.

- Biopsia hepática: biopsia hepática guiada por ecografía en el lóbulo derecho hepático, con una aguja Tru cut de 14 G (Allegiance Healthcare, Ill). Se obtuvieron dos especímenes. Uno se fijó durante 12-24 horas en formaldehído tamponado al $4 \%$ y se procesó de forma rutinaria para estudio histológico: se utilizaron las tinciones hematoxilina-eosina, tricrómico de Masson y Perls azul Prusia. La fibrosis fue estadiada utilizando un método semicuantitativo -F0: no fibrosis; F1: tractos portales ligeramente ensanchados; F2: fibrosis periportal; F3: fibrosis en puentes porto-portales; F4: cirrosis- $(13,14)$. Fibrosis leve F0-2; fibrosis avanzada F3-4. La otra muestra fue secada, pesada, y enviada para medición de la $\mathrm{CHH}$ por espectrofotometría (Laboratorio Reference. Barcelona, España).

-Mediciones por RM: RM de 1.5 Teslas (Philips Intera, Osatek. San Sebastián, España), para medir CHH por RM. Se obtuvieron dos secuencias eco-gradiente (T2, IW), siguiendo el método de Alustiza y cols. $(15,16)$. Tras ello se midió la intensidad de señal (IS): se midieron en cada secuencia tres mediciones de IS en el lóbulo derecho hepático y dos en los músculos sacroespinales. El ratio de IS hígado/músculo se calculó en cada secuencia. La CHH se cuantificó siguiendo el modelo matemático previamente publicado (15).

- Análisis estadístico: las variables predictivas fueron analizadas con los puntos de corte previamente determinados (9-13), y se calcularon sensibilidad, especificidad, VPP y VPN, con sus intervalos de confianza.

\section{RESULTADOS}

Estudio retrospectivo de 36 pacientes con $\mathrm{HH}$ y expresión fenotípica. Treinta y dos cumplieron los criterios de inclusión previamente publicados $(10,12,14)$. Tres fueron excluidos por $\mathrm{VHC}(+)$ y otro por no cumplir criterios de CHH/índice de hierro hepático. Siete mujeres $(21,8 \%)$ y 25 hombres $(78,1 \%$ ). Edad media 47,42 (rango 23-70). Las características clínicas y demográficas se presentan como media \pm desviación estándar y rango (Tabla I). Treinta y un pacientes tenían estudio HFE: 14 homocigotos C282Y $(45,16 \%), 5$ C282Y/H63D (16,16\%), 3 homocigotos H63D $(9,67 \%)$. El resto tenía genotipos no relacionados con enfermedad. En 7 pacientes se realizó RM con determinación de $\mathrm{CHH}$ siguiendo el protocolo de Alustiza y cols. $(15,16)$. La biopsia hepática reveló F0-2

Tabla I. Características clínicas/datos de laboratorio

\begin{tabular}{lr}
\hline & Media (rango) \\
\hline Edad & $47,42(23-70)$ \\
Sexo (mujer/hombre) & $7 / 25$ \\
Ferritina $(\mu \mathrm{g} / \mathrm{l})$ & $943,59(294-3.530)$ \\
Transferrina $(\%)$ & $64,09(30-104,6)$ \\
AST $(\mathrm{U} / \mathrm{L})$ & $37,11(17-211)$ \\
ALT $(\mathrm{U} / \mathrm{L})$ & $43,06(18-466)$ \\
Bilirubina $(\mathrm{mg} / \mathrm{dl})$ & $0,78(0,4-2,37)$ \\
Plaquetas $\left(\times 10^{3} / \mathrm{ml}\right)$ & $192.370(83.000-294.000)$ \\
CHH $(\mu \mathrm{mol} / \mathrm{g})$ & $131,46(54,8-546)$ \\
Índice hierro hepático & $4,97(1,57-30)$ \\
\hline
\end{tabular}


en 23 pacientes; 9 pacientes tuvieron un grado de fibrosis F3-4. Los pacientes con HH y fibrosis avanzada eran predominantemente de mayor edad y con saturación de transferrina, hierro y ferritina séricas más elevadas (Tabla II). El índice de fibrosis mostró un diagnóstico correcto para fibrosis leve $(\mathrm{F} \leq 2)$ en 15 de los 23 , y en 7 de los 9 con fibrosis avanzada. En los 4 pacientes con F4, el índice de fibrosis fue $>480.000$ y las plaquetas $<200.000$. Los resultados de los diferentes métodos no invasivos analizados, además del índice de fibrosis por biopsia, se muestran en la tabla III.

Tabla II. Características de los pacientes con fibrosis leve y avanzada

\begin{tabular}{lcc}
\hline & $\begin{array}{c}\text { Fibrosis leve } \\
\text { media } \pm D S\end{array}$ & $\begin{array}{c}\text { Fibrosis avanzada } \\
\text { media } \pm \text { DS }\end{array}$ \\
\hline Ferritina & $793,79 \pm 391,79$ & $854,29 \pm 131,70$ \\
Plaquetas & $216.000 \pm 34.199$ & $128.802 \pm 71.395$ \\
Hierro & $173 \pm 47$ & $187,59 \pm 30,99$ \\
Transferrina \% & $58,01 \pm 18,6$ & $68,37 \pm 23,05$ \\
Edad & $42,31 \pm 12,13$ & $49,10 \pm 10,5$ \\
\hline
\end{tabular}

Tabla III. Resultados de los diferentes métodos analizados

\begin{tabular}{lcccc}
\hline & Sensibilidad & Especificidad & VPP & VPN \\
\hline Indice fibrosis (en biopsia) & $85,7 \%$ & $68 \%$ & $42,8 \%$ & $94,4 \%$ \\
& $(79,6-91,8)$ & $(59,8-76,2)$ & $(34,1-51,5)$ & $(90,4-98,4)$ \\
Plaquetas (<200.000) & $85,7 \%$ & $78,3 \%$ & $46,1 \%$ & $94,7 \%$ \\
& $(79,6-91,8)$ & $(71,1-85,5)$ & $(37,3-54,9)$ & $(90,8-98,6)$ \\
AST elevación (> 40 U/I) & $83,3 \%$ & $80,9 \%$ & $55,5 \%$ & $94,4 \%$ \\
& $(76,7-89,9)$ & $(74-87,8)$ & $(46,7-64,2)$ & $(90,4-98,6)$ \\
Ferritina (> 1.000) & $42,8 \%$ & $50 \%$ & $20 \%$ & $75 \%$ \\
& $(34,1-51,5)$ & $(41,2-58,8)$ & $(13-27)$ & $(67,4-82,6)$ \\
IF por RM (CHH x edad) & $66 \%$ & $100 \%$ & $100 \%$ & $80 \%$ \\
& $(48,1-83,9)$ & & & $(64,9-95,1)$ \\
\hline
\end{tabular}

Finalmente, analizamos la combinación de los diferentes métodos, obteniendo los mejores resultados con la asociación del índice de fibrosis, por biopsia o por RM, con las transaminasas, y las plaquetas con los valores de las transaminasas, con un VPN del $100 \%$ en ambas combinaciones.

\section{DISCUSIÓN}

En los últimos 10 años se han publicado diferentes métodos no invasivos para la predicción de fibrosis en $\mathrm{HH}$ (9-13). Guyader y cols. (9) revisaron las variables clínicas y analíticas de 197 pacientes con $\mathrm{HH}$, homocigotos C282Y, en Francia. Sus hallazgos fueron validados en un grupo de 113 pacientes de Canadá. Ningún paciente tuvo fibrosis avanzada si no presentaba hepatomegalia, elevación de AST, o ferritina $>1.000 \mathrm{ng} / \mathrm{ml}$. En un estudio en 66 pacientes homocigotos $\mathrm{C} 282 \mathrm{Y}$ en EE. UU. (11) se ob- servó que la edad era un elemento importante y que no existía fibrosis avanzada en pacientes menores de 40 años. Más recientemente, Beaton y cols. (10) estudiaron las variables no invasivas para predicción de cirrosis en 193 pacientes homocigotos C282Y en Canadá y fue validado en un grupo de 162 pacientes de Francia. La combinación de una ferritina $>1.000$, plaquetas $<200.000$ y elevación de AST diagnosticó correctamente la presencia de cirrosis en el 77\% de los pacientes canadienses y en el $90 \%$ de los franceses. Morrison y cols. (12) demostraron en un estudio multicéntrico en EE. UU. con pacientes con hemocromatosis fenotípica, que una ferritina sérica $<1.000$ hacía improbable la presencia de cirrosis, independientemente de la edad de los pacientes o de los valores de transaminasas, evitando la necesidad de una biopsia hepática con finalidad pronóstica en estos pacientes. En 2005, Olynyk y cols. (13) demostraron que la duración de la exposición al hierro por el hígado incrementa el riesgo de fibrosis significativa en $\mathrm{HH}$, dando un importante papel a la edad de los pacientes en la predicción de fibrosis. El producto de la edad y de la $\mathrm{CHH}$ (índice de fibrosis), obtenido por biopsia o RM, con un punto de corte de 480.000, obtuvo una sensibilidad del $100 \%$ y un $86 \%$ de especificidad para fibrosis avanzada (F3-4) $(13,14)$. Esto fue validado externamente por nuestro grupo (17).

Con este estado de evidencia, decidimos analizar en nuestros pacientes con $\mathrm{HH}$ y expresión fenotípica los diferentes parámetros clínico-analíticos previamente estudiados, incluyendo 4 variables predictoras (plaquetas, transaminasas, ferritina, índice de fibrosis) que habían demostrado resultados favorables en análisis previos. No incluimos la hepatomegalia debido a su imprecisión y difícil reproducción de resultados (subjetivos) en la exploración clínica (12). El estudio del gen HFE reveló un $45 \%$ de homocigotos $\mathrm{C} 282$ Y. Este bajo resultado puede explicarse por dos hechos: el bajo porcentaje de homocigotos en el País Vasco, tal como ha sido publicado previamente $(4,18)$, y a los cambios en los algoritmos diagnósticos $(1,8,19,20)$, evitando en los últimos 5 años las biopsias de los pacientes con $\mathrm{HH}$ con genotipos típicos $\mathrm{y}$ datos clínico-analíticos que descartan fibrosis avanzada, y por lo tanto no pudiendo ser incluidos según los criterios de inclusión.

Un importante resultado es que en nuestra serie los niveles de ferritina (> 1.000) sólo tuvieron un 20\% de VPP, lo que claramente difiere de otros estudios en otras poblaciones, no permitiendo en nuestros pacientes evitar la biopsia hepática para fines pronósticos.

Las plaquetas y el índice de fibrosis demostraron ser las mejores variables para descartar fibrosis avanzada (Tabla III) con un VPN del 94\%. La determinación de la CHH por RM está siendo cada vez más reconocida como un valioso método $(21,22)$, y el índice de fibrosis ha demostrado ser útil cuando se calcula por biopsia o por RM $(13,17)$. En nuestra serie el índice de fibrosis por RM demostró también buenos resultados (especificidad 100\%; VPN $80 \%$ ). 
Finalmente hemos analizado la combinación de las diferentes variables, obteniendo los mejores resultados con la asociación del índice de fibrosis por biopsia o RM y las plaquetas, con las cifras de transaminasas, obteniendo un $100 \%$ de VPN para predicción de fibrosis de alto grado con ambas combinaciones.

En conclusión, en nuestro estudio la duración de la exposición hepática al hierro es importante en el desarrollo de fibrosis en $\mathrm{HH}$, tal como había sido publicado por Olynyk y cols. (13). El índice de fibrosis $>480.000$ y las plaquetas $<200.000$ tienen la mayor sensibilidad para la predicción de fibrosis de alto grado. Su resultado negativo nos permite descartar la presencia de fibrosis significativa en el 94\% de los casos. La RM con determinación de CHH en el País Vasco (15) permite obtener una buena predicción de fibrosis. Los valores de ferritina no muestran resultados tan buenos en nuestra serie. Aunque se requieren nuevos estudios con más pacientes para confirmar estos resultados, parece una realidad atractiva y no lejana el poder diagnosticar y predecir fibrosis en $\mathrm{HH}$ sin utilizar métodos invasivos.

\section{BIBLIOGRAFÍA}

1. Adams PC. Review article: the modern diagnosis and management of haemochromatosis. Alim Pharmacol Ther 2006; 23: 1681-91.

2. Beaton MD, Adams PC. The myths and realities of hemochromatosis. Can J Gastroenterol 2007; 21: 101-4.

3. Feder JN, Gnirke A, Thomas W, et al. A novel MHC class I-like gene is mutated in patients with hereditary haemochromatosis. Nat Genet 1996; 13: 399-408.

4. De Juan MD, Reta A, Castiella A, et al. HFE gene mutations analysis in Basque hereditary hemochromatosis patients and controls. Eur J Hum Genet 2001; 9: 961-4.

5. O'Neil J, Powell L. Clinical aspects of hemochromatosis. Sem Liv Dis 2005; 25: 381-91.

6. Beutler E. Penetrance in hereditary hemochromatosis. The HFE Cys282Tyr mutation as a necessary but not sufficient cause of clinical hereditary hemochromatosis. Blood 2003; 101: 3347-50.

7. Vázquez Romero M, Boixeda de Miquel D, Martin de Argila de Prados C, Vallcorba Gómez del Valle J, Cabello Albendea P, López San Román A, et al. Familiar penetrancy of HFE gene: four brothers of the same family affected by hereditary hemochromatosis. Rev Esp Enferm Dig 2005; 97: 608-9.

8. Pietrangelo A. Hereditary hemochromatosis - A new look to an old disease. N Engl J Med 2004; 350: 2383-97.

9. Guyader D, Jaquelinet C, Moirand R, et al. Noninvasive prediction of fibrosis in $\mathrm{C} 282 \mathrm{Y}$ homozygous hemochromatosis. Gastroenterology 1998; 115: 929-36.

10. Beaton M, Guyader D, Deugnier Y, Moirand R, Chakrabarti S, Adams P. Noninvasive prediction of cirrhosis in C282Y-linked hemochromatosis. Hepatology 2002; 36: 673-8.

11. Bacon BR, Olynyk JK, Brunt EM, Britton RS, Wolff RK. HFE genotype in patients with hemochromatosis and other liver diseases. Ann Intern Med 1999; 130: 953-62.

12. Morrison ED, Brandhagen DJ, Phatak PD, et al. Serum ferritin level predicts advanced hepatic fibrosis among U.S. patients with phenotypic hemochromatosis. Ann Intern Med 2003; 138: 627-33.

13. Olynyk JK, St Pierre TG, Britton RS, Brunt EM, Bacon BR. Duration of hepatic iron exposure increases the risk of significant fibrosis in hereditary hemochromatosis: A new role for magnetic resonance imaging. Am J Gastroenterol 2005; 100: 837-41.

14. Brunt EM. Pathology of hepatic iron overload. Sem Liv Dis 2005; 25: 392-401.

15. Alustiza JM, Artetxe J, Castiella A, et al. MR quantification of hepatic iron concentration. Radiology 2004; 230: 479-84.

16. Alustiza JM, Castiella A, De Juan MD, et al. Iron overload in the 1iver: diagnostic and quantification. Eur J Radiol 2007; 61: 499-506.

17. Castiella A, Emparanza JI. External validation for fibrosis predicting index in hereditary hemochromatosis. Am J Gastroenterol 2005; 100: 2366-7.

18. Bauduer F, Scribans C, Degioanni A, Renoux M, Dutour O. Distribution of the $\mathrm{C} 282 \mathrm{Y}$ and H63D polymorphisms in hereditary hemochromatosis patients from the French Basque Country. Ann Hematol 2005; 84: 99-102.

19. EASL Internacional Consensus Conference on Haemochromatosis. J Hepatol 2000; 33: 485-504.

20. Tavill AS. Diagnosis and management of hemochromatosis. Hepatology 2001; 33: 1321-8.

21. Aguilar-Martinez P, Schved JF, Brissot P. The evaluation of hyperferritinemia: an updated strategy based on advances in detecting genetic abnormalities. Am J Gastroenterol 2005; 100: 1185-94.

22. Castiella A, Alustiza JM, Artetxe J. Hereditary hemochromatosis. N Eng1 J Med 2004; 35: 1263-4. 